\title{
Acknowledgement to Reviewers of Algorithms in 2016
}

\author{
Algorithms Editorial Office \\ Published: 10 January 2017 \\ MDPI AG, St. Alban-Anlage 66, 4052 Basel, Switzerland; algorithms@mdpi.com
}

The editors of Algorithms would like to express their sincere gratitude to the following reviewers for assessing manuscripts in 2016.

We greatly appreciate the contribution of expert reviewers, which is crucial to the journal's editorial process. We aim to recognize reviewer contributions through several mechanisms, of which the annual publication of reviewer names is one. Reviewers receive a voucher entitling them to a discount on their next MDPI publication and can download a certificate of recognition directly from our submission system. Additionally, reviewers can sign up to the service Publons (https://publons.com) to receive recognition. Of course, in these initiatives we are careful not to compromise reviewer confidentiality. Many reviewers see their work as a voluntary and often unseen part of their role as researchers. We are grateful to the time reviewers donate to our journals and the contribution they make.

If you are interested in becoming a reviewer for Algorithms, see the link at the bottom of the webpage http://www.mdpi.com/reviewers.

The following reviewed for Algorithms in 2016:

Abu-Khzam, Faisal N.

Afshar-Nadjafi, Behrouz

Agrawal, Ankit

Aguero, Juan-Carlos

Aguilar, Sergio Ríos

Aker, Ahmet

Akhtar, Zahid

Alavi, Amir H.

Albergante, Luca

Ali, Irfan

Alonso, Pedro

Amirshahi, Seyed Ali

Amundarain, Aiert

Andreoni, Giuseppe

Angelov, Krasimir

Argenti, Fabrizio

Argyros, I.K.

Argyros, Ioannis

Asahiro, Yuichi

Azeloglu, Evren U

Badinelli, Ralph

Baiocchi, Valerio

Balaprakash, Prasanna

Behringer, Reinhold

Benítez, Héctor
Benítez López, Julio

Berretti, Stefano

Bhatia, Kanwal

Bianca, Carlo

Bienkowski, Marcin

Binucci, Carla

Borkowski, Piotr

Boulle, Marc

Branco, Rodrigo G.

Cain, Stephen

Cambria, Erik

Cao, Huiping

Cattani, Carlo

Ceci, Michelangelo

Cerello, Piergiorgio

Chang, Yao-Tang

Chatterjee, Shre Kumar

Chau, K.W.

Chen, Chih-Yuan

Chen, Shyi-Ming

Chen, Yangjun

Chiang, Tsung-Che

Chih, Mingchang

Ching, Wai-ki

Chuang, Cheng-Long
Cocconcelli, Marco

Comai, Sara

Companys, Ramon

Costello, James

Croce, Danilo

Cui, Changcai

Davies, Claire

De Cursi, E. Souza

De la Sen, Manuel

De Meo, Pasquale

De Oliveira Oliveira, Mateus

Degli Agosti, Robert

Del Mastio, Andrea

Delmastro, Franca

Demo Souza, Richard

Deng, Peng

Dengel, Andreas

Déniz , $O$.

Dewasme, Laurent

Donnarumma, Francesco

Dounis, Anastasios I.

DOWNEY, Rodney Graham

Duru, Kenneth

Dyke, Shirley

Emmerich, Michael 
Ennaji, Abdellatif

Feng, Tao

Fenu, Gianni

Ferrara, Massimiliano

Fiannaca, Antonino

Fiore, Marco

Fjordholm, Ulrik Skre

Florencio, Christophe Costa

Foti, Dora

Fried, Isaac

Froelich, Wojciech

Frontoni, Emanuele

Gajic, Zoran

Gao, Xiang

García Díaz, Pilar

Geem, Zong Woo

Gelvez, Nancy

Gendron, Paul

Ghim, Cheol-Min

Gildea, Daniel

Gil-Lopez, Sergio

Giuliani, Alessandro

Gkizeli, Maria

Gomez-Gonzalez, M.

Gong, Haijun

González G, David

Gonzalez-Pardo, Antonio

Granat, Janusz

Grigoriev, Alexander

Grimmer, Matthias

Gromov, Vasilii A.

Guccione, Pietro

Gui, Guan

Guilloux, Agathe

Hardy, Simon V.

Hashemi, Javad

Hassani, Hossein

Hayashida, Morihiro

He, Jing

He, Yanyan

He, Yue-Jing

Heiner, Monika

Henriques, Rui

Herisanu, Nicolae

Hernandez Perdomo, Wilmar

Herrich, Markus

Hodge, Victoria

Hong, Wei-Chiang

Howle, Victoria

Huang, Mao Lin

Huang, Weizhang

Hung, Ho-Lung
Iglesia, Didac Gil De La

Iglesias, Eva Lorenzo

Iliyasu, Abdullah M.

Irace, Zacharie

Iyiola, Olaniyi Samuel

Jaiswal, J. P.

Jayavelu, Senthilnath.

Jeinsch, Torsten

Jung, Peter

Jünger, Michael

Kaklamani, Dimitra

Kang, Myeongsu

Kantardzic, Mehmed M.

Karasozen, Bulent

Kari, Jarkko

Karyotis, Vasileios

Kauranne, Tuomo

Kawase, Yasushi

Kayacan, Erdal

Kelly, Deborah F.

Kesselheim, Thomas

Khodabandeh, Amir

Kiang, Jean-Fu

Kien, Le Trung

Kindermann, Philipp

Kiritchenko, Svetlana

Ko, Chia-Nan

Konstantinou, Ioannis

Kontogiannis, Spyros

Kotsiantis, Sotiris

Kratzke, Nane

Kriksciuniene, Dalia

Kulkarni, Anand Jayant

Kuremoto, Takashi

Kvasov, Dmitri E.

Kwok, Ngai Ming

Landa-Torres, Itziar

Langone, Rocco

Le Rouzic, Arnaud

Lee, Chao-Hsien

Lee, Cheng-Ming

Lee, Chun-Liang

Lee, Inbok

Lee, Yang-han

Li, Simon

Li, Yingsong

Liao, Xuejun

Lin, Chun-Cheng

Lin, Hongwei

Lin, Yuan-Pin

Lio, Yuhlong

Liu, Jia
Liu, Ya-Jie

Lizcano, David

Lopes, António M.

Luque, Gabriel

Ma, Yide

Machado, Maria Augusta

Soares

Magnien, Clemence

Magreñán, Ángel

Maletti, Andreas

Manjarrés, Diana

Manlove, David

Mannini, Andrea

Marian, Romeo

Marschall, Tobias

Martalò, Marco

Martinelli, Luca

Mashwani, Wali Khan

Mathews, Dave

Matuszyk, Paweł

Maurino, Andrea

Menendez, Hector

Meo, Marianna

Mercas, Robert

Mercorelli, Paolo

Meyer-Nieberg, Silja

Mezzini, Mauro

Mielikainen, Jarno

Migallon, $\mathrm{H}$.

Miyazaki, Shuichi

Mohammad, Abd El Khalick

Montanaro, Ashley

Morabito, Francesco Carlo

Morrison, Matthew

Mourtzis, Dimitris

Mousa, Amr El-Desoky

Muhlethaler, Paul

Mukhopadhyay, Asish

Murrugarra, David

Mzyk, Grzegorz

Naik, Ganesh R.

Nakas, Christos

Naldi, Aurélien

Namiot, Dmitry

Nehorai, Arye

Nevat, Ido

Nourelfath, Mustapha

Novelli, Antonio

Nuchkrua, Thanana

Odaira, Rei

Okhotin, Alexander

Olsen, Catharina 
Oneto, Luca

Ong, Benjamin W.

Orsenigo, Carlotta

Ortigosa, Pilar Martínez

Ouyang, Chen-Sen

Paja, Wieslaw

Pandey, Mayank

Pandit, Manjaree

Papa, Joao Paulo

Papavasiliou, Anastasia

Pasluosta, Cristian

Patrignani, Maurizio

Patwary, Md. Mostofa Ali

Pedersen, Marius

Perez-Uribe, Andres

Petra, Stefania

Pettersson, William

Pirola, Yuri

Polimeno, Antonino

Polyakov, Andrey

Popescu, Vlad

Porcelli, Margherita

Portillo Rodríguez, Otniel

Prochniewicz, Dominik

Qin, Zhiwei (Tony)

Quessy, Jean-François

Quilliot, Alain

Rademaker, Alexandre

Radhakrishnan, Chandra

Ramanathan, Parmesh

Ramos, Fernando M. V.

Recupero, Diego Reforgiato

Reddy, Yenumula

Reich, Simeon

Ricolfe-Viala, Carlos

Rinaldi, Franca

Risi, Michele

Rivenson, Yair

Rivera, Francisco F.

Rodger, James A.
Rodrigues, M. M.

Rong, Hongbo

Rossi, Luca

Rucco, Matteo

Sadakane, Kunihiko

Saddolah, Ali

Safacas, Athanasios

Salazar-Torres, Jose J.

Samborski, Sylwester

Schmid, Markus L.

Schütze, Oliver

Seetan, Raed

Sekhposyan, Tatevik

Sergeyev, Yaroslav

Serpedin, Erchin

Serra-Capizzano, Stefano

Shapira, Dana

Shojafar, Mohammad

Smyth, W. F.

Solomonik, Edgar

Song, Shiji

Steinley, Douglas

Stone, John E.

Stramer, Osnat

$\mathrm{Su}, \mathrm{Te}$-Jen

Sukhov, Vladimir

Szabo, Alexandre

Tabei, Yasuo

Tagliaferri, Francesca

Takaoka, Tadao

Takes, Frank

Taravat, Alireza

Tarhio, Jorma

Toivanen, Miika

Tsagkatakis, Grigorios

Tsai, Cheng-Mu

Tsai, Chun-Wei

Tsikerdekis, Michael

Udanor, Collins

Ullmann, R. Thomas
Uschmajew, André

Van Dinh, Anh

Vasilakos, Athanasios V.

Volpe, Gualtiero

Vörös, Jozef

$\mathrm{Vu}$, Ngoc Thang

Wang, Dong

Wang, Gai-Ge

Wang, Jianzhong

Wang, Lei

Wang, Yu

Wedekind, Jürgen

Wen, Kun-li

Wildey, Tim

Will, Sebastian

Wimmer, Christian

Wisniewski, Remigiusz

Wu, Jiunn-Lin

Wu, Jui-Yu

Wu, Qingbiao

Xiao, Robert

Xiong, Jiping

$\mathrm{Xu}$, Zeshui

Yamakami, Akebo

YANG, Le

Yang, Xin-She

Yen, Hsu-Chun

Yeoh, Phee

$\mathrm{Yu}$, Xiao-Hua

Zabkowski, Tomasz

Zamani, Mazdak

Zeng, Xueying

Zhan, Junpeng

Zhang, Guangming

Zhang, Haopeng

Zhang, Shihua

Zhang, Yudong

Zhao, Minbo

Zhuang, Hanqi

(C) 2017 by the authors; licensee MDPI, Basel, Switzerland. This article is an open access article distributed under the terms and conditions of the Creative Commons Attribution (CC-BY) license (http://creativecommons.org/licenses/by/4.0/). 